\title{
Facteurs déterminants la malnutrition aiguë sévère avec complication chez les enfants âgés de moins de 5 ans dans le district sanitaire de Banamba, Mali, 2014-2018
}

\section{Determinants of severe acute malnutrition with complications among children under 5 in the Banamba health district, Mali, 2014-2018}

\author{
Dembélé $\mathrm{I}^{1}$, Sogodogo $\mathrm{S}^{2}$, Kay \\ 1. Direction Régionale Santé, Koulikoro, Mali \\ 2. Inspection de la Santé, Ministère de la Santé et de l'Hygiène \\ Publique \\ 3. African Field Epidemiology Network, DGSHP, Bamako, Mali \\ 4. DER des Sciences Biologiques et Médicales, FAPH, USTTB, \\ Bamako, Mali \\ 5. Département d'Enseignement et de Recherche en Santé Publique, \\ FMOS, USTTB, Bamako, Mali \\ 6. Direction Générale de la Santé et de l'Hygiène Publique (DGSHP), \\ Bamako, Mali \\ 7. Direction Régionale Santé, Sikasso, Mali
}

Auteur correspondant : Dr Issiaka Dembélé, Direction Régionale Santé, Koulikoro, Email : dembeleissiaka2002@yahoo.fr, dembeleissiaka03@gmail.com, Tél : 76146698 / 66650695

\section{Résumé}

Introduction : Le district de Banamba a enregistré un taux de morbidité de malnutrition de $13 \%$ lié à la malnutrition. Cette étude avait pour objectif d'identifier les facteurs déterminants de la survenue de la malnutrition aiguë sévère avec complication chez les enfants âgés de moins de cinq dans le district sanitaire de Banamba au Mali. Méthodologie : il s'agissait d'une étude transversale rétrospective déroulée en octobre 2019. Les données du 01er janvier 2014 au 31 décembre 2018 ont été collectées. L'analyse a été faite avec Epi info 7.2 et Excel 2013 avec réalisation du test Exact Fisher et présentation des rapports de prévalences en plus des résultats descriptifs. Résultats : Nous avons enregistré au total 171 cas de malnutrition dont 10 cas $(5,85 \%)$ de kwashiorkor et 161 cas $(94,15 \%)$ de marasme. La quasitotalité $(93,57 \%)$ provenait du milieu rural. Le sex-ratio était de 1,04 . Plus de la moitié $(56,72 \%)$ avait entre 6 à 23 mois. Presque toutes les mères étaient mariées $(94,15 \%)$ et ménagères $(95,32 \%)$. Peu d'entre elles $(8,19 \%)$ étaient scolarisées. Le sevrage précoce a été beaucoup pratiqué avec $94,15 \%$. L'admission à l'Unité de Récupération et d'Education Nutritionnelle Intensive (URENI) a été spontanée pour la grande majorité soit 96,91\%. L'allaitement n'était pas exclusif presque toutes les femmes avec 99,42\%. Aucune association significative n'a été trouvée entre les facteurs et la malnutrition aiguë sévère avec complication. Conclusion: Notre étude a concerné 171 enfants malnutris avec complication. Les facteurs sexe masculin, lieu de résidence rurale, profession ménagère et mères non scolarisées étaient majoritaire mais n'étaient pas associés à la survenue des formes cliniques de la malnutrition avec complication.

Mots clés: déterminants, kwashiorkor, marasme, Banamba, Mali. baly $\mathrm{CA}^{4}$, Telly $\mathrm{N}^{4}$, Koné $\mathrm{Y}^{6}$, Diakité $\mathrm{S}^{7}$, Traoré $\mathrm{B}^{3}$

Abstract

Introduction: The district of Banamba recorded a malnutrition morbidity rate of $13 \%$ linked to malnutrition. The objective of this study was to identify the factors associated with the occurrence of severe and complicated malnutrition in children under the age of five in the Banamba health district in Mali. Methodology: It was a retrospective cross-sectional held in October 2019. Data collection from January 1, 2014 to December 31, 2018 was collected. Analysis was done using Epi info 7.2 and Excel 2013 software. We present descriptive results and analytics one using Fisher Exact test and prevalence ratio. Results: We recorded a total of 171 cases of malnutrition including 10 cases $(5.85 \%)$ of kwashiorkor and 161 cases $(94.15 \%)$ of marasmus. Almost all $(93.57 \%)$ came from rural areas. The sex ratio was 1.04 . more than half $(56.72 \%)$ were between 6 and 23 months old. Almost all the mothers were married $(94.15 \%)$ and housewives $(95.32 \%)$. Few of them $(8.19 \%)$ were in school. Early weaning was widely practiced with $94.15 \%$. Admission to Intensive Nutritional Recovery and Education Unit (URENI) was spontaneous for the vast majority $(96.91 \%)$. Breastfeeding was not exclusive for almost all women with $99.42 \%$. No significant association was found between the factors and severe acute malnutrition Conclusion: Our study concerned 171 malnourished children with complications. Male factors, place of rural residence, housework and mothers who were not in school were in the majority, but were not associated with the occurrence of clinical forms of malnutrition with complications.

Keywords: determinants, kwashiorkor, marasmus, Banamba, Mali.

\section{Introduction}

La malnutrition est un ensemble de manifestations dues à un apport inadéquat en quantité et / ou en qualité dans l'alimentation de substances nutritives nécessaires à la croissance normale et au bon fonctionnement de l'organisme (1).

Dans le monde 143 millions d'enfants de moins de 5 ans souffrent de malnutrition, dont 20 millions de Malnutrition Aiguë Sévère (MAS). Chaque minute, environ 10 enfants malnutris meurent, soit près de 5 millions chaque année. La malnutrition représentait au moins $30 \%$ de la mortalité infantile en 2013 (2). Elle reste un véritable problème de santé publique dans la plupart des pays en développement. En 2014, le taux de MAS était estimé à 
9.8\% dans la Région Africaine de l'Organisation Mondiale de la Santé (OMS) (2).

Sur la plan national, les taux de prévalence obtenus en $2015,2016,2017$ sont de $12,4 \% ; 10,7 \%$ et $10,7 \%$ pour la Malnutrition Aiguë Globale (MAG), pour la Malnutrition Aiguë Modérée (MAM) 9,6\%;8,6\%; $8,1 \%$ et de 2,8\% ; $2,1 \% ; 2,6 \%$ pour la Malnutrition Aiguë Sévère (MAS) (3). Dans la région de Koulikoro, les taux de prévalence obtenus en 2015, 2016, 2017, sont respectivement de $11,2 \% ; 10,6 \% ; 7,8 \%$ et de $9,2 \% ; 9,1 \% ; 6,5 \%$ et de $2,0 \% ; 1,5 \% ; 1,3 \%$ (3). Situé dans la région de Koulikoro, le district sanitaire de Banamba a enregistré un taux de morbidité liée à la malnutrition aiguë de $13 \%$, avec un taux de décès de $0,27 \%$ lié au MAS sans complications. Aussi 67 cas de malnutrition sévère avec complication ont été notifiés (3). C'est dans ce contexte que se situe notre étude dont l'objectif était d'identifier les facteurs déterminants de la survenue de la malnutrition aiguë sévère avec complication chez les enfants âgés de moins de cinq dans le district sanitaire de Banamba au Mali.

\section{Méthodologie}

Cadre d'étude :

Notre travail s'est déroulé au niveau de la de région de Koulikoro dans le district sanitaire de Banamba couvrant une superficie d'environ $7200 \mathrm{Km}^{2}$ avec 261953 habitants en 2019 , on compte 20 CSComs fonctionnels, 3 cabinets médicaux, 9 officines de pharmacie repartis entre 9 communes rurales. Le district est limité par les circonscriptions de Nara au Nord, Koulikoro au Sud, Niono et Ségou à l'Est, Kolokani à l'Ouest.

Type d'étude :

II s'agissait d'une étude transversale rétrospective qui s'est déroulée en octobre 2019

Période d'étude :

L'étude s'est déroulée en octobre 2019. Elle a porté sur les données de malnutrition aiguë sévère avec complication de la période janvier 2014 au 31 décembre 2018.

\section{Critère d'inclusion}

L'ensemble des cas de la malnutrition aiguë sévère avec complication de mois de 5 ans admis à l'URENI du CSRéf de Banamba quelques soient la mesure du périmètre brachial et la pathologie associée du 01er janvier2014 au 31 décembre 2018.

\section{Critères de non inclusion}

Tous cas de malnutrition aiguë sévère avec une complication de moins de 5 ans ayant déjà séjourné une première fois dans une URENI.

\section{Taille de l'échantillon et échantillonnage}

En prenant une précision $5 \%$ et un risque d'erreur de $5 \%$, la taille de l'échantillon est donnée par la formule de SCHWARTZ :

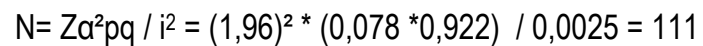

$Z a=1,96$

i= précision $=0,05$

$p=0.078 \quad q=0.922$
L'URENI du CSRéf de Banamba est la seule structure concernée et l'ensemble des cas répondants aux critères d'inclusion soit 171 cas de malnutrition aiguë sévère avec complication.

Techniques et outils de collectes des données

Revue documentaire, fiche individuelle de prise en charge et registre de l'URENI

Cette étude s'est déroulée en deux phases, d'une part à la collecte des données qui a consisté à exploiter les registres de consultations de prise en charge des malnutritions avec complications du CSRéf de l'unité de récupération nutritionnelle intensive(URENI) de Banamba et des fiches individuelles de chaque malades et d'autre part à la saisie, traitement et analyse des données sur Epi info 7.2, Excel. Les données ont été analysées pour ressortir les caractéristiques qui corroborent avec les objectifs spécifiques, le Test Exact Fisher pour rechercher les liens entre variables et rapport de prévalence pour mesurer la force de l'association.

\section{Considérations éthiques}

Le respect strict, absolu de la confidentialité des informations et l'anonymat des dossiers ont été assurés.

\section{Résultats}

Nous avons enregistré au total 171 cas de malnutrition dont 10 cas $(5,85 \%)$ de kwashiorkor et 161 cas $(94,15 \%)$ de marasme. Le plus grand nombre de cas de MAS avec complication a été enregistré en 2018 soit $39,18 \%$. La majorité des cas provenait du milieu rural soit 93,57\%. Nous avons noté une prédominance masculine avec un sex-ratio de 1,04. Les enfants de 6 à 23 mois étaient les plus touchés soit $56,72 \%$. La majorité des mères était mariée avec $94,15 \%$ avec comme principales occupations des travaux ménagers soit 95,32\%. Seulement $8,19 \%$ des femmes étaient scolarisées. Le mode de sevrage précoce était majoritaire soit $94,15 \%$. Le mode d'admission spontané était majoritaire soit 96,91\%. Le mode d'allaitement non exclusif était majoritaire avec $99,42 \%$ des cas.

A l'analyse bivariée (tableau I), nous n'avons pas noté de différence de fréquences de marasme entre les deux sexes $(p=0,48)$. L'intervalle inter génésique inférieur à 24 mois semblait moins atteint par le marasme. II n'y avait pas de différence de prévalence entre les tranche d'âge $(p=0,46)$. Les enfants du milieu rural étaient plus susceptibles au marasme que ceux du milieu urbain mais sans relation significative. Le mode d'allaitement non exclusif a été le plus représenté soit 160 cas sans différence significative observée. Tous les enfants des femmes célibataires souffraient de marasme sans différence significative comparée à ceux des mariées $(p=0,27)$. La profession ménagère a été le plus représentée mais aussi dont les enfants avaient beaucoup plus souffert de marasme mais significativité $(p=0,23)$. Ce constat était pareil pour les enfants des femmes non scolarisés et des enfants non sevrés précocement. 


\section{Discussion}

Limite de l'étude: la taille de l'échantillon des cas de kwashiorkor était très faible. Cette faible a du influé la significativité de nos tests d'association. En effet aucun de nos tests n'était significatif.

Description en temps, lieu et personne

Ce travail nous a permis d'évaluer 171 cas de malnutrition aiguë sévère avec complications. La majorité des cas provenait du milieu rural soit $93,57 \%$. Une prédominance masculine a été observée dans notre étude comme dans celle Ouédraogo SO. au Burkina en 2012 et de Diop M. au CSRéf de Markala en 2015 avec un sex ratio respectivement de 1,54 (4) et 1,2 (5). II était différent des résultats de Diarra $\mathrm{N}$. au CSRéf de la commune I de Bamako qui trouve une prédominance féminine avec un sex ratio de 0,9 (6).

Notre étude avait révélé que les enfants de 6 à 23 mois étaient les plus touchés par la malnutrition aiguë sévère soit $56,72 \%$. II était légèrement différent à celle de Diop M. qui trouve $69 \%$ (5). La quasi-totalité des mères enquêtées était mariée et ménagères soient $95,32 \%$ des femmes. Notre résultat était supérieur à celui de Savadogo AS dans la région de Ségou qui trouve $88,0 \%$ (7). Seulement $8,19 \%$ des femmes ont été scolarisées. Ce taux était inférieur à celui de Doumbia MN en 2001 au CHU Gabriel TOURE qui trouve 18,6\%(8). Chez les enfants de notre étude, la majorité avait eu un sevrage précoce et n'avait pas bénéficié d'allaitement exclusif. II s'agit de facteur qui concoure non seulement à la malnutrition aiguë mais aussi à ses complications dont le marasme qui était le plus retrouvé chez la quasi-totalité et le kwashiorkor. Le marasme serait la forme de malnutrition aiguë sévère le plus répandu dans le district sanitaire de Banamba. Cette prédominance a été également notée au service de pédiatrie de l'hôpital de Gao en 2009 par Barry BO. qui trouve $54,4 \%$ (9) et Diarra I. au CSRéf de Koutiala en 2015 qui trouve 50,5\% (10).

Identification des facteurs déterminants de la survenue de la malnutrition

Les enfants de sexe masculin couraient 1,01 fois plus de risque de développer la malnutrition aiguë que les enfants de sexe féminin sans différence significative observée $(p=0,48)$. L'intervalle inter génésique inférieur à 24 mois a été le plus représenté soit 141 cas sans différence significative observée $(p=0,43)$. La tranche d'âge de 0 à 23 mois a été la plus représentée soit 124 cas sans différence significative observée $(p=0,46)$. Les enfants provenant du milieu rural avaient 1,04 fois plus de risque de développer la malnutrition aiguë que les enfants du milieu urbain sans différence significative observée $(p=0,31)$. Le mode d'allaitement non exclusif a été le plus représenté soit 160 cas sans différence significative observée $(p=0,47)$. Le statut matrimonial des mères mariées a été le plus représenté soit 151 cas sans différence significative observée $(p=0,27)$. Les enfants des mères ménagères avaient 1,08 plus de risque de développer la malnutrition aiguë que les enfants des mères sans différence significative observée $(p=0,23)$. Les mères non scolarisées couraient 1,02 plus de risque de développer la malnutrition aiguë que les mères scolarisées sans différence significative observée $(p=0,39)$. Le mode de sevrage précoce a été le plus représenté soit 151 cas sans différence significative observée $(p=0,27)$.

\section{Conclusion}

Notre étude a concerné 171 enfants malnutris avec complication. Les facteurs sexe masculin, lieu de résidence rurale, profession ménagère et mères non scolarisées étaient des facteurs déterminants majoritaires mais non significativement associés à la survenue de la malnutrition aiguë sévère avec complication dont le marasme et le kwashiorkor. Cependant, il y a lieu de sensibiliser les mères sur l'alimentation du nourrisson et du jeune enfant notamment sur les méthodes de sevrage et l'allaitement exclusif.

\section{Références}

1. Protocole_PECIMA_Mali 23_06-2012VF.pdf [Internet]. [cité 2 oct 2019]. Disponible sur: https://www.humanitarianresponse.info/sites/www.human itarianresponse.info/files/documents/files/Protocole_PECl MA_Mali\%2023_06-2012VF.pdf

2. UNICEF. Malnutrition aiguë sévère [Internet]. UNICEF. [cité 2 oct 2019]. Disponible sur: https://www.unicef.org/french/nutrition/index_sam.html

3. Kanto Andriamanjatoson. Un enfant sur trois souffre de malnutrition dans le monde [Internet]. Daily Geek Show. 2019 [cité 30 oct 2019]. Disponiblesur: https://dailygeekshow.com/un-enfant-sur-trois-souffremalnutrition-monde/

4. OMS | Prise en charge de la malnutrition aiguë sévère chez les enfants de 6 à 59 mois présentant un œdème [Internet]. WHO. [cité 2 oct 2019]. Disponible sur: http://www.who.int/elena/titles/oedema_sam/fr/

6. Traoré FM. Aspects épidemio-cliniques de la malnutrition aigue sévère des enfants de moins de 5 ans au CHU.GT. 2014 [cité 2 oct 2019]; Disponible sur: https://www.bibliosante.ml/handle/123456789/637

7. Ouédraogo S, Yugbaré $E, A L$. Facteurs de risque de mortalité au cours de la malnutrition aiguë sévère dans le service de pédiatrie du Centre Hospitalier régional (CHR) de Kaya, Article ID C120104, 6 pages doi:10.4303/cmch/C120104 4(1) : 85-90. :6.

8. Diop M. Evaluation de la prise en charge en charge de la malnutrition aiguë sévère dans I'URENI du CSREF de MARKALA en 2014, thèse de Médecine, Faculté de Médecine et d'Odontostomatologie de 
I'Université des Sciences, des Techniques et des Technologies de Bamako. 2015;82.

9. Diarra N. Les infections courantes dans le cas de la malnutrition aiguë sévère des enfants de 6 à 59 mois dans le service de pédiatrie du CSRéf de la commune I du district de Bamako. Thèse de médecine, Faculté de Médecine et d'Odontostomatologie de l'Université des Sciences, des Techniques et des Technologies de Bamako. 2014;89.

Tableau I : Analyse bivariée : relation entre les facteurs déterminants et les formes cliniques de la malnutrition aiguë sévère

\begin{tabular}{|c|c|c|c|c|c|}
\hline facteurs déterminants & Marasme & Kwashiorkor & $\%$ marasme & $\mathrm{RP}[\mathrm{IC95 \%}]$ & $p$ \\
\hline \multicolumn{6}{|l|}{ Sexe } \\
\hline Masculin & 95 & 6 & 95,06 & $1,01[0,19-4,22]$ & 0,48 \\
\hline Féminin & 66 & 4 & 94,29 & & \\
\hline \multicolumn{6}{|l|}{ Intervalle inter génésique } \\
\hline Inférieur à 24 mois & 141 & 10 & 93,38 & $0,98[0,01-5,77]$ & 0,43 \\
\hline Supérieur ou égale 24 mois & 19 & 1 & 95,00 & & \\
\hline \multicolumn{6}{|l|}{ Tranche d'âge } \\
\hline $0-23$ mois & 124 & 8 & 93,94 & $0,99[0,08-4,64]$ & 0,46 \\
\hline $24-59$ mois & 37 & 2 & 94,87 & & \\
\hline \multicolumn{6}{|l|}{ Lieu de résidence } \\
\hline Rural & 151 & 8 & 94,38 & $1,04[0,03-14,45]$ & 0,31 \\
\hline Urbain & 10 & 1 & 90,91 & & \\
\hline \multicolumn{6}{|l|}{ Mode d'allaitement } \\
\hline Non exclusif & 160 & 10 & 94,12 & $0,94[0,91-0,98]$ & 0,47 \\
\hline Exclusif & 1 & 0 & 100,00 & & \\
\hline \multicolumn{6}{|l|}{ Statut matrimonial mère } \\
\hline Mariée & 151 & 10 & 93,79 & $0,94[0,00-7,91]$ & 0,27 \\
\hline Célibataire & 10 & 0 & 100,00 & & \\
\hline \multicolumn{6}{|l|}{ Profession de la mère } \\
\hline Ménagère & 154 & 9 & 94,48 & $1,08[0,83-1,41]$ & 0,39 \\
\hline Salariée & 7 & 1 & 87,5 & & \\
\hline \multicolumn{6}{|l|}{ Niveau d'étude de la mère } \\
\hline Non Scolarisée & 148 & 9 & 94,27 & $1,02[0,87-1,18]$ & 0,39 \\
\hline Scolarisée & 13 & 1 & 92,86 & & \\
\hline \multicolumn{6}{|l|}{ Mode de sevrage } \\
\hline Précoce & 151 & 10 & 93,79 & $0,94[0,90-0,98]$ & 0,27 \\
\hline Non précoce & 10 & 0 & 100,00 & & \\
\hline
\end{tabular}

